\title{
Impaired JAK-STAT signal transduction contributes to growth hormone resistance in chronic uremia
}

\author{
Franz Schaefer, ${ }^{1,2}$ Yu Chen, ${ }^{1}$ Tanny Tsao, ${ }^{1}$ Pouneh Nouri, ${ }^{1}$ and Ralph Rabkin ${ }^{1}$ \\ ${ }^{1}$ Research Service, Veterans Affairs Palo Alto Health Care System, and Department of Medicine, Stanford University, \\ Palo Alto, California, USA \\ ${ }^{2}$ Division of Pediatric Nephrology, University Children's Hospital, Heidelberg, Germany \\ Address correspondence to: Ralph Rabkin, Veterans Affairs Palo Alto Health Care System, 111R, 3801 Miranda Avenue, \\ Palo Alto, California 94304, USA. Phone: (650) 858-3985; Fax: (650) 849-0213; E-mail: rabkin@stanford.edu.
}

Received for publication December 1, 2000, and accepted in revised form June 22, 2001.

\begin{abstract}
Chronic renal failure (CRF) is associated with resistance to the growth-promoting and anabolic actions of growth hormone (GH). In rats with CRF induced by partial renal ablation, 7 days of GH treatment had a diminished effect on weight gain and hepatic IGF-1 and IGFBP-1 mRNA levels, compared with sham-operated pair-fed controls. To assess whether GH resistance might be due to altered signal transduction, activation of the JAK-STAT pathway was studied 10 or 15 minutes after intravenous injection of $5 \mathrm{mg} / \mathrm{kg} \mathrm{GH}$ or vehicle. Hepatic GH receptor (GHR) mRNA levels were significantly decreased in CRF, but GHR protein abundance and GH binding to microsomal and plasma membranes was unaltered. JAK2, STAT1, STAT3, and STAT5 protein abundance was also unchanged. However, GH-induced tyrosine phosphorylation of JAK2, STAT5, and STAT3 was $75 \%$ lower in the CRF animals. Phosphorylated STAT5 and STAT3 were also diminished in nuclear extracts. The expression of the suppressor of cytokine signaling-2 (SOCS-2) was increased twofold in GH-treated CRF animals, and SOCS-3 mRNA levels were elevated by $60 \%$ in CRF, independent of GH treatment. In conclusion, CRF causes a postreceptor defect in GH signal transduction characterized by impaired phosphorylation and nuclear translocation of GH-activated STAT proteins, which is possibly mediated, at least in part, by overexpression of SOCS proteins.
\end{abstract}

J. Clin. Invest. 108:467-475 (2001). DOI:10.1172/JCI200111895.

\section{Introduction}

Growth failure is a major complication of chronic renal failure (CRF) (1). Children with CRF are partially resistant to the growth-promoting effect of growth hormone $(\mathrm{GH})$, and the response to endogenous $\mathrm{GH}$ and pharmacological GH treatment are impaired in proportion to the degree of renal failure. Partial GH resistance has been reproduced in the uremic rat (2), where hepatic IGF-1 gene expression fails to increase in response to $\mathrm{GH}(3)$.

Several mechanisms of GH resistance in uremia have been suggested, including reduced $\mathrm{GH}$ receptor (GHR) gene expression $(4,5)$, increased IGF- 1 binding to accumulated serum-binding proteins (6), and a postreceptor defect in IGF-1 action (7), though the latter is not a uniform finding (8). Moreover, since GHR gene expression is reduced less than IGF-1 gene expression (5) and GHR binding is similar to pair-fed controls in uremic rats (9), the failure of GH to stimulate IGF-I gene expression suggests the possibility that a postreceptor defect in GH signaling may also be present.

Recently, the intracellular signaling pathways activated by GH have been elucidated (10). Binding of GH to its membrane receptor induces dimerization of the receptor, resulting in tyrosine phosphorylation of Janus-associated kinase 2 (JAK2), a tyrosine kinase associated with the intracellular domain of the receptor. Phosphorylation induces the kinase activity of
JAK2, which in turn phosphorylates a group of molecules known as signal transducers and activators of transcription (STATs). The GHR-JAK2 complex activates mainly STAT5 and to a lesser degree STAT3 and STAT1. Upon phosphorylation, the STATs form dimers that translocate into the nucleus, bind to specific promoter sequences of GH-dependent genes, and transactivate or repress their transcription. Recently, a family of proteins has been identified that bind to cytokine receptor-JAK2 complexes and inhibit JAK2 kinase activity (11). Some of these suppressors of cytokine signaling (SOCS), namely SOCS-1, $-2,-3$, and CIS, are induced by GH via the JAK-STAT signaling pathway and inhibit the GH-induced activation of the GHR-JAK2 complex (11-13), constituting an intracellular feedback loop regulating GH sensitivity.

The importance of the JAK-STAT signaling pathway for postnatal growth has been demonstrated in selective gene-knockout models. Male mice with STAT5b deficiency and female mice with a combined deletion of STAT5a and STAT5b isoforms are severely growth retarded $(14,15)$, whereas SOCS-2-knockout animals develop gigantism (16). It is therefore conceivable that alterations of the JAK-STAT signaling pathway might be involved in the postulated postreceptor defect of $\mathrm{GH}$ action in CRF. In this study, we tested this postulate by evaluating hepatic $\mathrm{GH}$ signal transduction in CRF rats with evidence of $\mathrm{GH}$ resistance. 


\section{Methods}

\section{Experimental animals and protocols}

Pituitary-intact male SD rats weighing 100-120 g were allocated to CRF or pair-fed (PF) control groups. CRF was induced by a two-stage five-sixth nephrectomy procedure. In the first intervention, approximately two-thirds of the left kidney was excised through a flank incision. Seven days later, the right kidney was removed, subsequently resulting in a state of stable CRF. In control animals, a sham procedure (exposure of kidney) was performed. Control animals received the amount of food consumed by the CRF animals on the previous day (pair feeding).

GH treatment study. Two weeks after the second surgical intervention, CRF and PF control animals were subdivided into two groups receiving either twice daily subcutaneous injections of recombinant human $\mathrm{GH}(\mathrm{hGH})$ (Genentech Inc., South San Francisco, California, USA) at a dose of $10 \mathrm{mg} / \mathrm{kg} /$ day or $\mathrm{V}$ (vehicle) for 7 days. This dose was selected based on pilot studies in which lower doses of GH, 1 and $5 \mathrm{mg} / \mathrm{kg} /$ day, failed to stimulate body growth and reflects the relative insensitivity of the pituitary-intact rat to exogenous $\mathrm{GH}$ compared with hypophysectomized rats (17). On the 7 th day, the rats were sacrificed 5-6 hours after the last GH or V injection. Blood was collected by aortic puncture, and blood gas analysis was performed immediately. Sera were separated and kept at $-20^{\circ} \mathrm{C}$. The liver was quickly removed, snap-frozen in liquid nitrogen, and stored at $-80^{\circ} \mathrm{C}$.

GH-signaling study. CRF and PF control animals were studied 2 weeks after establishing CRF. The animals were anaesthetized, and for these signaling studies, a bolus of recombinant bovine $\mathrm{GH}(\mathrm{bGH})(5 \mathrm{mg} / \mathrm{kg})$ (Monsanto Corp., St. Louis, Missouri, USA) or V was injected intravenously. In one set of experiments, a small piece of liver was ligated and removed for basal analysis immediately before, and the remaining liver was collected 15 minutes after the $\mathrm{GH}$ injection. In another series of experiments in which the rats were fasted overnight, the initial liver biopsy was omitted, and whole liver was collected 10 minutes after the intravenous administration of $\mathrm{GH}$ or $\mathrm{V}$, snap-frozen, and stored at $-80^{\circ} \mathrm{C}$. A pharmacological dose of $\mathrm{GH}$ was used based on the pilot studies noted earlier. Indeed, pharmacological doses are commonly used in studies of hormone resistance because this ensures that differences between animals are not due to impaired hormone delivery (18). Finally, high-dose GH therapy more closely reflects the clinical situation where children with CRF are treated with pharmacological doses of GH (1).

In pilot studies with rats fed ad libitum, the levels of phosphorylated STAT5 in animals not treated with GH were variable and often elevated, reflecting the pulsatile nature of GH secretion. However, in CRF rats and their PF controls, basal phospho-STAT5 was undetectable or very low, reflecting the known suppressive effect of reduced food intake on endogenous $\mathrm{GH}$ secretion in rats with and without CRF (19). Accordingly, the study was restricted to CRF rats and PF controls.
Biochemical measurements and IGF-1 radioimmunoassay

Serum creatinine was measured with a Creatinine Analyzer (Beckman Coulter Inc., Palo Alto, California, USA) and tissue protein content by the Bradford method (Protein Assay Kit; Bio-Rad Laboratories Inc., Hercules, California, USA). Serum IGF-I concentrations were determined in acid-ethanol extracts using a radioimmunoassay kit (Nichols Institute Reference Laboratories, San Juan Capistrano, California, USA).

\section{Preparation of liver membranes}

Crude and purified liver plasma membranes were prepared by modifications of published protocols $(20,21)$. Briefly, crude membranes were obtained by homogenizing $0.5 \mathrm{~g}$ of liver in $0.25 \mathrm{M}$ ice-cold sucrose at $20,000 \mathrm{rpm}$ using an Ultra-Turrax homogenizer (IKA-LAB ortechnik, Staufen, Germany). After centrifugation at $1,000 \mathrm{~g}$ for 10 minutes, the supernatant was centrifuged at $100,000 \mathrm{~g}$ for 60 minutes at $4^{\circ} \mathrm{C}$. The pellet containing crude liver membranes was resuspended with $0.25 \mathrm{M}$ sucrose. For purification of plasma membranes, 2-3 g of liver were homogenized in cold buffer (ST buffer) containing 0.25 M sucrose and $10 \mathrm{mM}$ Tris- $\mathrm{HCl}(\mathrm{pH} 7.5)$. The homogenate was diluted, centrifuged at $1,500 \mathrm{~g}$ for 15 minutes, and resuspended in a self-forming Percollsucrose gradient. Centrifugation for 20 minutes at $35,000 \mathrm{~g}$ resulted in a viscous layer containing plasma membranes and DNA. This layer was collected, rediluted, and centrifuged at 45,000 $\mathrm{g}$ for 30 minutes in a gradient containing Percoll, sucrose, and $\mathrm{CaCl}_{2}$. The plasma membrane layer was collected, rediluted in ST buffer, pelleted by centrifugation at $1,500 \mathrm{~g}$ for 30 minutes, and resuspended in ST buffer. The activity of $5^{\prime}$ - $\alpha$-nucleotidase, a plasma membrane marker enzyme used to determine plasma membrane enrichment, was assayed in the homogenates and crude and purified plasma membrane preparations as described previously (22).

\section{GHR binding assay}

GHR binding was measured both in crude and plasma membranes by a receptor binding assay as before (23). The bGH was radiolabeled by the Iodo-Gen method (Pierce Chemical Co., Rockford, Illinois, USA). Briefly, membrane aliquots were preincubated with $3 \mathrm{M} \mathrm{MgCl}_{2}$ for 5 minutes to remove endogenous prebound $\mathrm{GH}$ and then incubated with $10^{-11} \mathrm{M}\left[{ }^{125} \mathrm{I}\right]-\mathrm{GH}$ at $4{ }^{\circ} \mathrm{C}$ overnight. Free and liver-membrane receptor-bound $\left[{ }^{125} \mathrm{I}\right]-\mathrm{GH}$ were separated by centrifugation at $15,000 \mathrm{~g}$ for 10 minutes and quantitated in a gamma counter. Specific [ $\left.{ }^{125} \mathrm{I}\right]-\mathrm{GH}$ binding was determined by displacement with $10^{-6} \mathrm{M}$ unlabeled bGH

\section{Northern blot analysis}

The cDNA probes were prepared for the analysis of GHR, IGFBP-1, CIS, and SOCS-2 mRNA as before (23). The GHR probe was prepared from a $2.2-\mathrm{kb}$ full-length mouse cDNA kindly provided by F. Talamantes (University of California Santa Cruz, Santa Cruz, California, 
USA) (24). This cDNA encodes a sequence encompassing the extra- and intracellular domain of the receptor. The IGFBP-1 probe consisted of 407 bp corresponding to nucleotide positions 486 to 892 in the rat IGFBP-1 sequence (25). The cDNA probes for CIS (770 bp) and SOCS-2 (794 bp) were kindly provided by A. Yoshimura (Karume University, Karume, Japan) (11). The cDNA probes were labeled with $50 \mu \mathrm{Ci}\left[{ }^{32} \mathrm{P}\right]$-dCTP by a random primer method (Multiprime DNA labeling system, Amersham Biotech, Inc., Piscataway, New Jersey, USA) RNA was isolated from liver as described previously (23). Samples containing $20 \mu \mathrm{g}$ denatured RNA were loaded and electrophoresed on a denaturing agarose gel, transferred, and fixed by ultraviolet cross-linking to nitrocellulose filters, and prehybridized at $42^{\circ} \mathrm{C}$ for 4 hours as before (23). The mRNA levels of interest were detected by hybridizing ${ }^{32} \mathrm{P}$-labeled probes to mRNA at $42^{\circ} \mathrm{C}$ overnight. The filters were washed and the signal either measured directly in a PhosphorImager device using Image Quant software (Molecular Dynamics, Sunnyvale, California, USA), or after exposure of filters to Kodak XAR film (Eastman Kodak Co., Rochester, New York, USA) by densitometric analysis (Fluor-S device and MultiAnalyst Software; Bio-Rad Laboratories Inc.).

\section{Solution hybridization RNase protection assay}

Transcript-specific ${ }^{32} \mathrm{P}-\mathrm{CTP}$ labeled cRNA probes were transcribed in vitro from respective cDNA vector constructs. The IGF-1 probe, a 376-bp Sau3A-EcoRI fragment, was designed to detect both Ea and Eb IGF-1 mRNAs (26). The $18 \mathrm{~S}$ riboprobe contained 80 bases complementary to a highly conserved region of the human RNA (27). Rat-specific CIS, SOCS-2, and SOCS-3 cDNA was amplified by PCR, using primer pairs published previously (13). The PCR product was cloned into a TOPO TA plasmid (Invitrogen Corp., San Diego, California, USA) and in vitro transcribed and radiolabeled as described above. Twenty micrograms total RNA was hybridized with the radiolabeled antisense riboprobes at $42^{\circ} \mathrm{C}$ overnight. The mixture was then incubated with an RNase digestion buffer followed by the addition of proteinase K. Ethanol-precipitated protected hybrids were separated on a polyacrylamide/urea denaturing gel. Autoradiography was performed at $-20^{\circ} \mathrm{C}$ using an intensifying screen. Protected bands were quantitated densitometrically.

\section{Immunoprecipitation and Western immunoblot} analysis

Ab's against STAT1, STAT3, phospho-STAT1, and phospho-STAT3 were obtained from New England Biolabs Inc. (Beverly, Massachusetts, USA). The antiSTAT5 Ab that detects STAT5a and STAT5b, the antiphosphotyrosine ( $\alpha$-PY20) Ab, and protein A agarose were from Santa Cruz Biotechnology Inc. (Santa Cruz, California, USA). For the detection of phospho-STAT5, an $\mathrm{Ab}$ from Upstate Biotechnology Inc. (Lake Placid, New York, USA) was used. This polyclonal Ab detects the tyrosine-phosphorylated forms of both STAT5A and STAT5B. The GHR Ab mab263, raised against purified rat GH receptor (28), was purchased from Biogenesis Inc. (Kingston, New Hampshire, USA).

Frozen liver, $100 \mathrm{mg}$, was homogenized on ice with a Polytron (Brinkmann Instruments Inc., Westbury, New York, USA) at maximum speed for 30 seconds in 7 volumes of lysis buffer (100 mM Tris, $\mathrm{pH}$ 7.4, 1\% Triton X100) containing $100 \mathrm{mM}$ sodium pyrophosphate, 100 $\mathrm{mM} \mathrm{NaF}, 10 \mathrm{mM}$ EDTA, $10 \mathrm{mM} \mathrm{Na}_{3} \mathrm{VO}_{4}, 2 \mathrm{mM}$ PMSF, and $0.1 \mathrm{mg} / \mathrm{ml}$ aprotinin. After 30 minutes in a shaker at $4^{\circ} \mathrm{C}$, extracts were centrifuged for 30 minutes at $45,000 \mathrm{~g}$ and the supernatants collected for further analysis. For the detection of liver GHR, tissue was homogenized in a hot lysis buffer containing $1 \%$ SDS and $10 \mathrm{mM}$ Tris $(\mathrm{pH}$ 7.5) with added $0.2 \mathrm{mM}$ PMSF and $0.2 \mathrm{mM} \mathrm{Na}_{3} \mathrm{VO}_{4}$. Homogenates were boiled for 10 minutes and then centrifuged as described above. Nuclear extracts were obtained from $100 \mathrm{mg}$ frozen tissue aliquots using the NE-PER extraction kit (Pierce Chemical Co.). For JAK2 immunoprecipitation (IP), $5 \mathrm{mg}$ of protein was diluted to $1 \mathrm{ml}$ in IP buffer (containing $10 \mathrm{mM}$ Tris, $\mathrm{pH} 7.4,1 \%$ Triton X, $150 \mathrm{mM} \mathrm{NaCl}, 1 \mathrm{mM}$ EDTA, 1 mM EGTA, 0.2 $\mathrm{mM} \mathrm{Na}_{3} \mathrm{VO}_{4}, 0.2 \mathrm{mM}$ PMSF, and $0.5 \%$ Nonidet P-40) and incubated with $2 \mu \mathrm{g} \mathrm{JAK} 2 \mathrm{Ab}$ 's overnight at $4^{\circ} \mathrm{C}$. The immune complexes were then adsorbed to protein A agarose beads for 2 hours at $4^{\circ} \mathrm{C}$, washed 4 times by centrifugation, and resuspensed in IP buffer.

The JAK2 immunoprecipitates or $50 \mu \mathrm{g}$ of liver lysate (for direct assay of all other proteins) were heated in Laemmli buffer at $100^{\circ} \mathrm{C}$ for 5 minutes, electrophoresed on a $7.5 \%$ SDS polyacrylamide gel, and electroblotted onto nitrocellulose membranes. Blots were blocked for 1 hour in TBST buffer (10 mM Tris, $\mathrm{pH} 7.4,138 \mathrm{mM}$ $\mathrm{NaCl}, 0.05 \%$ Tween- 20 containing 3-5\% nonfat dehydrated milk or $1 \%$ BSA. Subsequently, the blots of the JAK-2 IP were incubated overnight at $4^{\circ} \mathrm{C}$ with Ab's against phosphotyrosine residues ( $\alpha$-PY20). The blots of the liver lysates were incubated with Ab's directed against GHR, JAK2, STAT1, STAT3, STAT5, phospho-STAT1, phospho-STAT3, or phospho-STAT5. After washing three times for 15 minutes in TBST, the blots were incubated with secondary anti-rabbit or anti-mouse Ab conjugated to horseradish peroxidase (Santa Cruz Biotechnology Inc.) for 1 hour at room temperature and then washed again three times. The signal on the filter was detected by enhanced chemiluminescence (ECL; Amersham Life Sciences Inc.) and exposure to Kodak XAR film (Eastman Kodak Co.). Protein expression was quantitated densitometrically with a Fluor-S digital image analyzer and Multianalyst software (Bio-Rad Laboratories Inc.). Relative density units refer to mean pixel density with local background subtraction.

\section{Data analysis and statistics}

PhosphorImager and autoradiographic readings of specific mRNAs were normalized for 18S rRNA signal strength and expressed as transcript/18S ratios unless indicated otherwise. Similarly, as indicated later, phosphorylated protein readings were normalized for the 
Table 1

Growth response and biochemical characteristics in rats with CRF and PF controls treated with human $\mathrm{GH}$ or $\mathrm{V}$ for 7 days

\begin{tabular}{lcccc}
\hline & \multicolumn{2}{c}{ PF control } & \multicolumn{2}{c}{ CRF } \\
& Vehicle & GH & Vehicle & GH \\
Weight at start of treatment $(\mathrm{g})$ & $179 \pm 17$ & $178 \pm 17$ & $178 \pm 5$ & $178 \pm 6$ \\
Weight change during treatment $(\mathrm{g})$ & $25.4 \pm 4.4^{\mathrm{A}}$ & $55 \pm 1.6^{\mathrm{B}}$ & $25.1 \pm 11.2^{\mathrm{A}}$ & $34.4 \pm 9.2^{\mathrm{A}}$ \\
Food intake $(\mathrm{g})$ & $96.9 \pm 0^{\mathrm{A}}$ & $108 \pm 0^{\mathrm{B}}$ & $96.9 \pm 19.5^{\mathrm{A}}$ & $108 \pm 11^{\mathrm{B}}$ \\
Food conversion ratio $(\mathrm{g} / \mathrm{g})$ & $0.26 \pm 0.04^{\mathrm{A}}$ & $0.51 \pm 0.02^{\mathrm{B}}$ & $0.26 \pm 0.13^{\mathrm{A}}$ & $0.32 \pm 0.07^{\mathrm{A}}$ \\
Serum creatinine $(\mathrm{mg} / \mathrm{dl})$ & $0.70 \pm 0.07^{\mathrm{A}}$ & $0.56 \pm 0.21^{\mathrm{A}}$ & $1.8 \pm 0.27^{\mathrm{B}}$ & $1.69 \pm 0.47^{\mathrm{B}}$ \\
Arterial pH & $7.39 \pm 0.02^{\mathrm{A}}$ & $7.38 \pm 0.01^{\mathrm{A}}$ & $7.42 \pm 0.09^{\mathrm{A}}$ & $7.42 \pm 0.06^{\mathrm{A}}$ \\
Plasma bicarbonate $(\mathrm{mmol} / \mathrm{l})$ & $29.5 \pm 0.7^{\mathrm{A}}$ & $28.5 \pm 2.1^{\mathrm{A}}$ & $31 \pm 1.2^{\mathrm{A}}$ & $31.8 \pm 2.2^{\mathrm{A}}$ \\
Serum IGF-1 $(\mathrm{ng} / \mathrm{ml})$ & $625 \pm 31^{\mathrm{A}}$ & $817 \pm 113^{\mathrm{A}}$ & $828 \pm 62^{\mathrm{A}}$ & $1104 \pm 69^{\mathrm{A}}$
\end{tabular}

Results are mean \pm SEM of five control and seven CRF rats per group. Serum creatinine represents mean of values obtained at start and end of treatment period; all other biochemical parameters were obtained at time of sacrifice. Superscript letters indicate differences between the groups within each row (Newman-Keuls test, $P<0.05)$; groups with common superscript letters do not differ from each other.

respective protein levels. The control group mean (vehicle-treated in GH treatment study) was assigned a value of $100 \%$, and individual values are expressed relative to this value. Data are given as mean \pm SEM. Data were checked for Gaussian distribution using the Kolmogorov-Smirnov test (Sigmastat; Jandel Scientific Software, San Rafael, California, USA). Two-tailed unpaired Student $t$ tests were applied for comparison of two normally distributed groups; comparisons between more than two normally distributed groups were made by oneway ANOVA followed by pairwise multiple comparison (Student-Newman-Keuls method). For non-Gaussian distributions the Kruskal-Wallis test, followed by allpairwise comparisons (Dunn's test), was used. $P$ values less than 0.05 were considered statistically significant.

\section{Results}

Biochemical characteristics and GH effect on body weight gain and food consumption. As seen in Table 1, the subtotal nephrectomy produced a threefold increase in serum creatinine levels, which were stable during the observation period (serum creatinine at 2 weeks, $1.66 \pm 0.18$ $\mathrm{mg} / \mathrm{dl}$; at 3 weeks, $1.74 \pm 0.37 \mathrm{mg} / \mathrm{dl}$ ). Resistance to $\mathrm{GH}$ therapy was evident in the CRF rats; average weight gain during $\mathrm{GH}$ treatment was diminished by $69 \%$ and food use (i.e., weight gain per unit weight of food consumed) by $75 \%$ compared with the PF control group. The relative weight gain in the GH-treated CRF animals was inversely correlated with serum creatinine levels $(r=-0.75, P<0.05)$. Total serum IGF-1 levels were lower in both CRF and PF control animals than in ad libitum-fed controls (data not shown), but did not differ between CRF and PF controls. Serum IGF-1 levels were slightly increased by GH treatment both in $\mathrm{PF}(P=0.14)$ and in CRF animals $(P=0.12)$.

Effect of GH on IGF-I and IGFBP-1 gene transcription. Resistance to GH in CRF was also evident with respect to the expression of its hepatic target genes IGF-I and IGFBP-1. The results of the measurements of the IGF-1 Ea (276 bp) transcript and the IGFBP-1 transcript are expressed in relative arbitrary units and are shown in Figure 1. Basal IGF-I mRNA levels in the V-treated group did not differ between control (IGF-I Ea [276 bp], $100 \pm 9$; IGF-I Ea [100 bp], $100 \pm 7$; IGF-I Eb, $100 \pm 23$ relative arbitrary units) and CRF animals (IGF-I Ea [276 bp], $103 \pm 15$; IGF-I Ea [100 bp], $91 \pm 8$; IGF-Eb, $129 \pm 38$, not significant [NS]). In the controls GH treatment increased IGF-I Ea (276 bp) mRNA by $79 \% \pm 19 \%(P<0.05)$, IGF-I Ea (100 bp) by $67 \% \pm 17 \%$ $(P<0.05)$, and IGF-Eb mRNA by $158 \% \pm 36 \%(P<0.01)$. In contrast, $\mathrm{GH}$ had no significant effect in the CRF animals (IGF-I Ea [276 bp], $-2 \% \pm 38 \%$; IGF-I Ea [100 bp], $+21 \% \pm 29 \%$; IGF-I Eb, $+22 \% \pm 58 \%$ ) (Figure 1a). The effect of GH on IGF-I mRNA levels differed significantly for the IGF-I Ea (276 bp) and the IGF-I Eb transcript $(P<0.05)$. Basal IGFBP-1 mRNA levels were similar in the $\mathrm{V}$-treated control and CRF animals. However, the response to GH differed quantitatively. In the controls GH treatment suppressed IGFBP-1 gene expression by $86 \% \pm 4 \%(P<0.05)$, while in the CRF group by
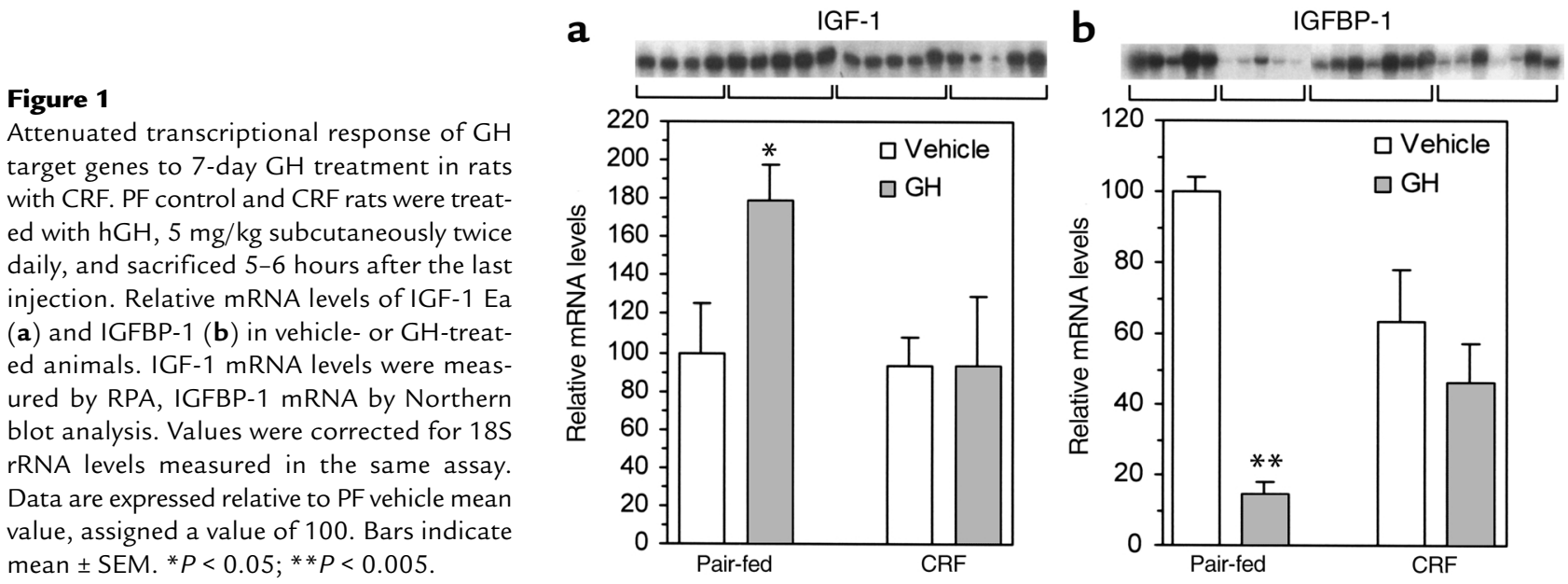
only $28 \% \pm 8 \%$ (Figure $1 \mathrm{~b}$ ). Relative suppression was significantly different between the two groups $(P<0.05)$. GHR gene expression, protein abundance, and binding capacity. Hepatic GHR mRNA levels were significantly reduced in the V-treated CRF animals of the 7-day GH treatment study (Figure 2a, $P<0.05$ ). Similarly, in the animals used for the GH signaling experiments, GHR mRNA levels were decreased by $61 \% \pm 4 \%$ compared with PF controls $(P<0.001)$. Chronic GH treatment did not change GHR gene expression in either the PF $(117 \% \pm 20 \%$ of PF-V), NS) or CRF group ( $113 \% \pm 11 \%$ of CRF-V, NS). In contrast to the difference in GHR mRNA levels, Western immunoblotting of the GHR protein showed a band around $130 \mathrm{kDa}$ that was equally abundant in the CRF and in the control animals in both studies (Figure 2b).

In addition to the analysis of GHR protein expression, measurements of specific GH binding to liver membranes were also performed. In the crude microsomal isolates, $\alpha-5^{\prime}$-nucleotidase activity was only slightly higher $(166 \pm 6 \mathrm{nmol} / \mathrm{mg}$ protein $/ \mathrm{min})$ than in native liver homogenates $(105 \pm 4 \mathrm{mmol} / \mathrm{mg} / \mathrm{min})$. In contrast, the mean $\alpha-5^{\prime}$-nucleotidase activity of the membranes obtained by Percoll gradient centrifugation was $1,451 \pm 134 \mathrm{mmol} / \mathrm{mg} /$ protein $/ \mathrm{min}$ vs. $77 \pm 19 \mathrm{nmol} / \mathrm{mg}$ protein $/ \mathrm{min}$ in homogenates, indicating a 22 -fold enrichment of plasma membranes by this procedure. Consistent with the results of Western immunoblot analysis, specific GH binding to either the crude microsomes or the purified plasma membranes did not differ between CRF animals and controls (Figure 2c).

JAK-STAT signal transduction. The protein levels of JAK2, STAT1, STAT3, and STAT5 in liver lysates were not different between CRF and control animals (Figure 3a). In contrast, differences in the level of phosphorylated proteins were evident after $\mathrm{GH}$ treatment (Figure $3 \mathrm{~b}$ ). In the basal state (V-treated animals), little phosphorylation of JAK2, STAT1, and STAT3, and no phosphorylation of STAT5, was detected in either group. JAK2 tyrosine phosphorylation increased 10 minutes after GH injection, but this response was blunted in the CRF animals (Figure 3, b and c). Similarly, GH-stimulated phosphorylation of STAT5 was $75 \%$ lower in CRF animals than in controls, as determined both by absolute signal strength $(P<0.0001)$ and when expressed relative to the corresponding level of STAT5 protein $(P<0.0001)$. In these experiments the $\mathrm{V}$ - and the GH-treated tissue was obtained from different animals. In another experiment (six animals per group), where tissue was obtained immediately before and 15 minutes after GH in the same animal, a similar reduction of STAT5 phosphorylation was observed (PF, $100 \pm 13$, vs. CRF, $31 \pm 6$ arbitrary units; $P<0.0005)$. A significant positive relationship between the relative phosphorylation of JAK2 and STAT5 after GH administration was observed $(r=0.87$; $P<0.001$ ) (Figure 3d). Regression analysis showed that relative STAT5 and JAK2 phosphorylation levels were inversely correlated with serum creatinine levels $(r=-0.91$, and $r=-0.89$, respectively, $P<0.0001)$.

Analogous differences between CRF and PF control animals were found with respect to STAT3 and STAT1 tyrosine phosphorylation. Absolute and relative phosphorylation of STAT3 were decreased in the CRF animals by $68 \%$ and $75 \%$, respectively (Figure $3, \mathrm{~b}$ and $\mathrm{c}$ ). The GH-induced STAT1 phosphorylation was very weak and more variable than the phospho-STAT3 and phospho-STAT5 signals, but also tended to be lower in the CRF animals (Figure 3, $\mathrm{b}$ and $\mathrm{c} ; P<0.087$ ).

To see whether the low whole-cell phospho-STAT levels were reflected in the nucleus, STAT phosphorylation was also measured in nuclear extracts 15 minutes after GH injection (Figure 4a). As in the whole cell, nuclear phospho-STAT5 was low in the CRF animals compared with the controls ( $38 \pm 22$ vs. $100 \pm 14$ arbitrary units, $P<0.05)$. Nuclear phospho-STAT3 levels were on average lower in the CRF group, but this did not reach statistical significance $(52 \pm 20$ vs. $100 \pm 19$ arbitrary units, $P=0.09$ ). Phospho-STAT1 was undetectable in the nuclear extracts both in CRF and control animals.

SOCS-2, CIS, and SOCS-3 gene expression. SOCS- 2 mRNA was detectable by Northern blot analysis only in animals that received prolonged GH treatment (Figure $4 \mathrm{~b}$ ). The 18S-corrected SOCS-2 mRNA levels were increased 2.5fold in the GH-treated CRF animals, compared with the GH-treated PF controls ( $250 \pm 41$ vs. $100 \pm 10$ arbitrary units, $P<0.005)$. As shown in Table 2 , the increased
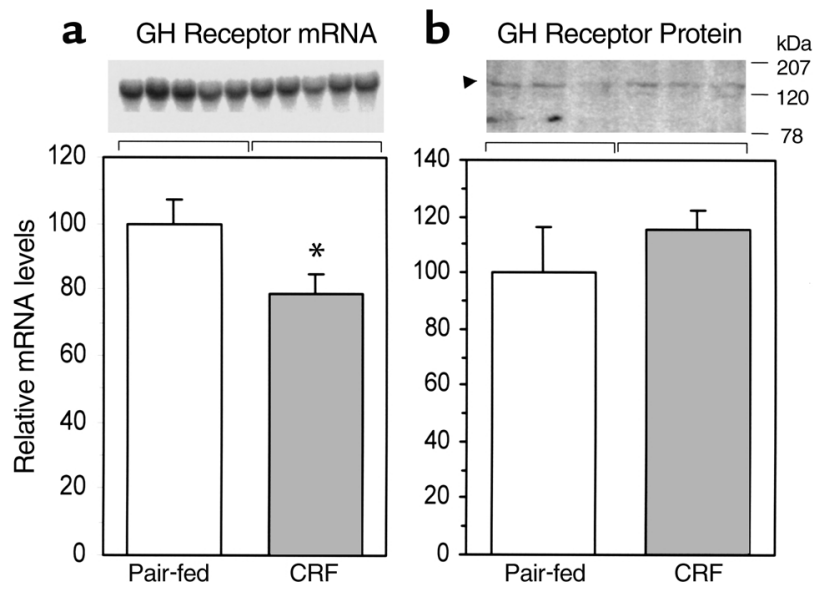

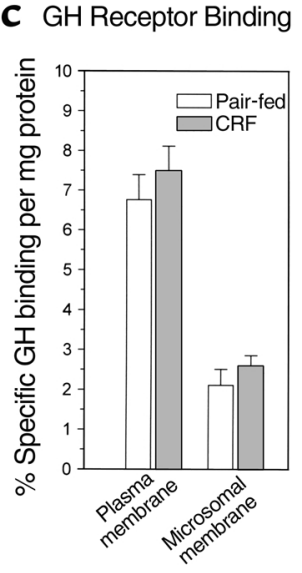

\section{Figure 2}

Hepatic GHR mRNA levels(a), but not protein abundance $(\mathbf{b})$ or $\mathrm{GH}$ membrane binding(c), are reduced in CRF. Hepatic GHR mRNA was measured by Northern blot analysis and GHR protein by Western immunoblot analysis. Specific binding of radiolabeled $\mathrm{bGH}$ to receptors in purified liver plasma membranes and microsomes was measured by displacement with an excess of unlabeled $\mathrm{bGH}$. All measurements were made in livers from the same animals. Data are expressed relative to PF vehicle-treated control mean value, assigned a value of 100 . Bars indicate mean \pm SEM. $n=4-6$ rats/group. ${ }^{*} P<0.05$.
\end{abstract}



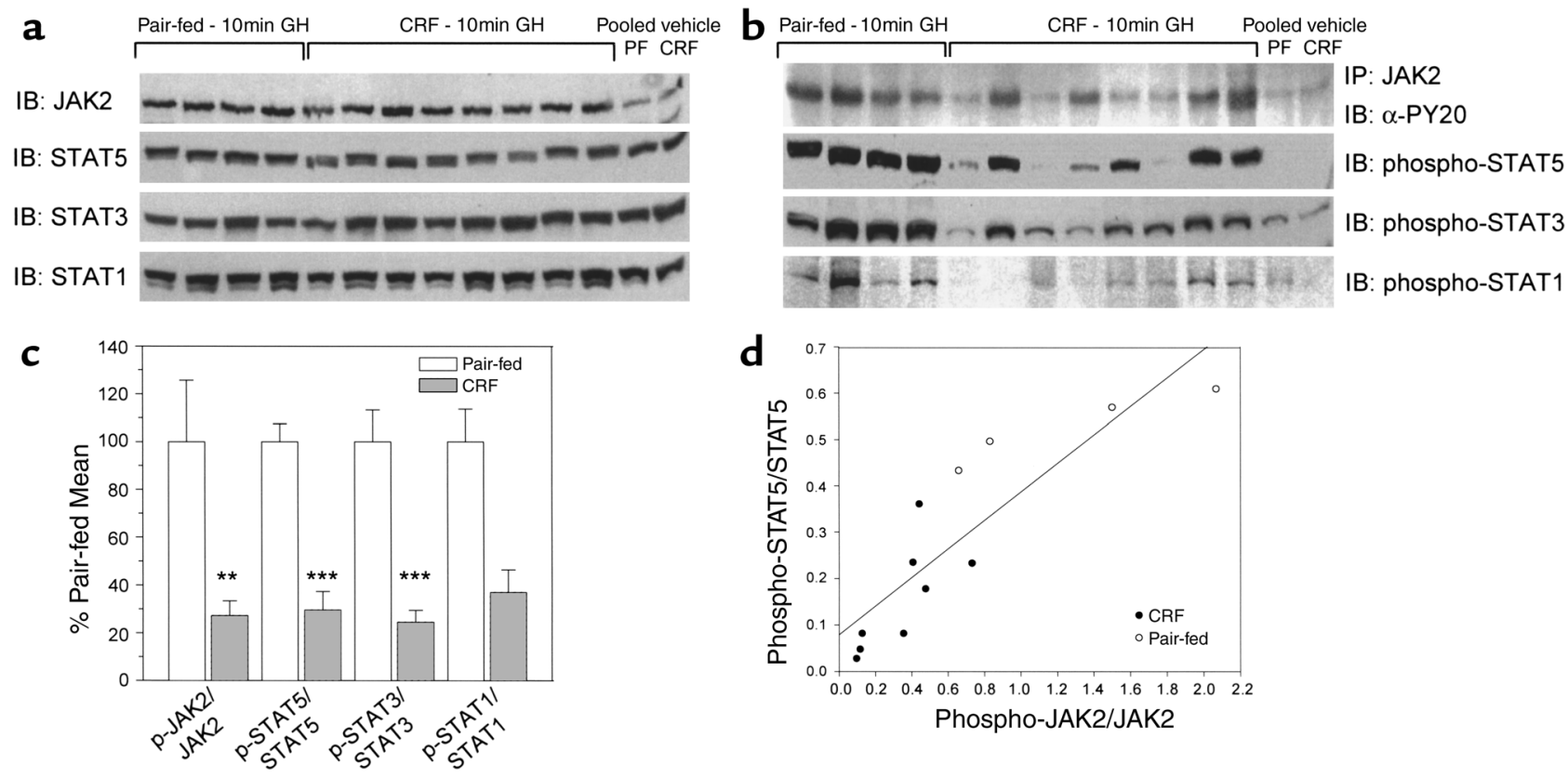

\begin{abstract}
Figure 3
Hepatic JAK2 and STAT5, STAT3, and STAT1 protein levels are unchanged in CRF, but protein tyrosine phosphorylation is impaired. (a) Western immunoblots of total JAK2, STAT1, STAT3, and STAT5 protein levels in liver lysates obtained from CRF and PF control animals 10 minutes after intravenous bGH, $5 \mathrm{mg} / \mathrm{kg}$, or vehicle. JAK2 and STAT proteins were measured after direct loading of lysate protein. The samples from each vehicle-treated group were pooled for this immunoblot. (b) Abundance of tyrosine-phosphorylated JAK and STAT proteins in the same samples as shown in a. Phospho-JAK2 was detected by immunoblotting JAK2 immunoprecipitates with antiphosphotyrosine Ab $(\alpha-P Y 20)$, In contrast, phospho-stats were detected without immunoprecipitation with phospho-specific Ab's. $n=4-6$ rats/group. (c) Relative phosphorylation of JAK2 and STAT proteins 10 minutes after bGH bolus in CRF and PF control animals. ${ }^{*} P<0.005$; ${ }^{* * *} P<0.0005$. Phospho-protein signals were corrected for the specific protein levels, and the ratios were normalized to the PF mean, which was assigned a value of 100. Bars indicate mean \pm SEM. (d) Relationship between relative phosphorylation levels of JAK2 and STAT5, each expressed as the ratio of phosphorylated/total specific protein signal.
\end{abstract}

SOCS-2 gene expression in GH-treated CRF animals was confirmed by RNase protection assay (RPA) $(909 \pm 137$ vs. $482 \pm 17$ arbitrary units, $P<0.01$ ). This sensitive assay also detected the SOCS-2 mRNA in the V-treated animals. Although on average higher in the CRF group, the levels were not significantly different from the PF control values because of the variability of basal expression. However bivariate ANOVA showed a significant GH-independent stimulatory effect of CRF on SOCS- 2 expression $(P<0.05)$. CIS mRNA expression, detectable only by RPA, was increased by GH treatment in the CRF and control animals $(P<0.0001)$. CIS expression tended to be higher in the CRF groups, but significance was not reached.

SOCS-3 mRNA levels, also only detectable by RPA, were not elevated in either group when measured 5-6 hours after the last GH injection, compared with $\mathrm{V}$ injection, a finding consistent with its rapid and transient treatment response (13). Accordingly the $\mathrm{V}$ - and $\mathrm{GH}$-treatment values were regarded as reflecting basal values and were pooled for analysis. This showed that the SOCS-3 mRNA levels were significantly higher in the CRF animals $(P<0.05$; Table 2$)$.

\section{Discussion}

In this study we set out to determine whether impaired GH-mediated signal transduction could contribute to the GH resistance that develops in CRF, and our results show that a defect in the JAK-STAT signal transduction pathway is indeed present in uremia. To evaluate GH signaling we used a rat model of nonacidotic CRF, severe enough to cause $\mathrm{GH}$ resistance. In these animals hepatic GHR binding and protein levels were similar to PF, shamoperated controls. Despite this, GH treatment failed to stimulate IGF-I gene expression, and, compared with the controls, suppression of IGFBP-1 gene expression and body weight gain were severely blunted. The key alterations in signal transduction uncovered were a diminution in GH-induced tyrosine phosphorylation of the GH receptor-associated tyrosine kinase JAK2 and reduced tyrosine phosphorylation and nuclear translocation of $\mathrm{GH}$-activated STAT proteins. We also found increased mRNA levels of the intracellular feedback regulatory proteins SOCS- 2 and SOCS- 3 in CRF rats that we propose may contribute to the impaired JAK-STAT signaling.

Previously, it has been suggested that GH resistance in CRF may be caused by a defect in GHR expression. Support for this has been provided by studies in uremic rats that showed reduced hepatic GHR mRNA levels. However, this reduction could in part be attributed to reduced food intake $(4,5)$. Indeed, others observed no difference in GHR mRNA levels between CRF and PF control animals $(9,29)$, questioning an independent 


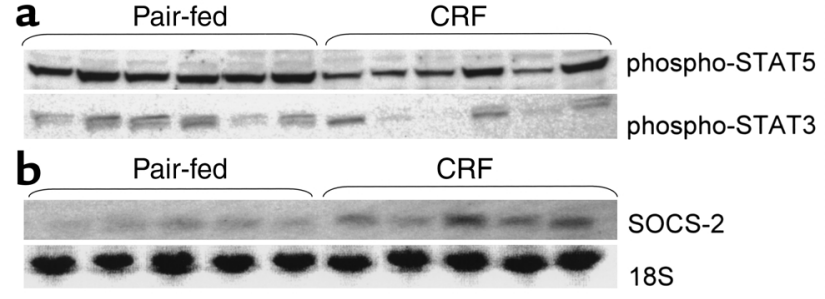

Figure 4

(a) Decreased nuclear accumulation of phosphorylated STATs in rats with CRF 15 minutes after $\mathrm{GH}$ administration. Intravenous bGH, 5 $\mathrm{mg} / \mathrm{kg}$, was given as a bolus. Western immunoblot analysis of nuclear protein extracts were performed using phosphotyrosine-specific Ab's against phospho-STAT5 and phosphoSTAT3. (b) Increased liver SOCS-2 mRNA levels in rats with CRF after 7 days of GH treatment. Subcutaneous hGH, $5 \mathrm{mg} / \mathrm{kg}$, was given twice daily, and the animals were sacrificed 5-6 hours after the final dose. SOCS-2 mRNA and $18 \mathrm{~S}$ rRNA levels were measured by Northern blot analysis. SOCS-2 mRNA was undetectable in the vehicle-treated rats with this assay.

suppressive effect of uremia. Similarly, whereas an early study suggested reduced GH binding to crude rat liver membranes in CRF compared with ad libitum-fed control animals (30), a later study showed that specific $\mathrm{GH}$ binding in CRF was not different from PF controls (9). On the other hand, reduced GHR protein expression has been found using immunohistochemistry in uremic rat growth cartilage (31). Clinical studies using circulating GH-binding protein levels, largely derived by cleavage of the extracellular GHR domain, as a surrogate marker of tissue GHR abundance in children with CRF have yielded conflicting results; both low (32) and normal GHBP plasma levels have been reported (33).

In this study we observed a significant reduction of steady-state GHR mRNA levels by $20-50 \%$, whereas GHR protein levels, measured by Western immunoblot analysis, were unchanged in the CRF rats compared with the PF controls. The reason for the disparity between the GHR mRNA and protein levels in the CRF animals was not examined, but appears to be due to a posttranscriptional event such as increased translational efficiency or decreased receptor protein degradation. To confirm our immunoblot findings and to exclude the possibility that resistance to $\mathrm{GH}$ is caused by a maldistribution of the GHR with a reduction in the number of plasma membrane receptors, we measured $\mathrm{GH}$ specific binding to crude microsomes and purified plasma membranes. Consistent with our measurements of GHR protein levels, receptor binding in both preparations was unaltered by $\mathrm{CRF}$. Hence, the GH resistance in the CRF animals cannot be attributed to an alteration in receptor number, affinity, or distribution and suggests a more distal defect.

The levels of the key-signaling proteins of the JAK/STAT signal-

Table 2 ing pathway in the liver, namely JAK2, STAT1, STAT3, and STAT5, were also unaffected by CRF. This left the possibility of diminished activation of these signaling molecules in response to GHR binding as a cause of the resistance. This hypothesis was tested by direct assessment of hepatic STAT tyrosine phosphorylation following a single intravenous GH bolus. Indeed, GH-induced STAT5 phosphorylation was suppressed by $70-75 \%$ in animals with CRF. Phosphorylated STAT5 was also low in nuclear cell extracts, indicating that the inhibition of STAT5 phosphorylation is severe enough to impair nuclear accumulation of phosphorylated STAT5 in response to GH. This, in turn, could be expected to result in depressed STAT5 transcriptional activity. GH also stimulated STAT3 and, to a small extent, STAT1 tyrosine phosphorylation, but the degree of phosphorylation was compromised in the CRF group to an extent comparable to that seen with STAT5. Phospho-STAT3 also tended to be low in the nucleus, suggesting that specific STAT3-mediated GH actions are also impaired in CRF.

As the tyrosine residues in the STAT molecules are exclusively phosphorylated by JAK2 in response to $\mathrm{GH}$, the deficient STAT phosphorylation in the presence of intact GHR protein levels strongly suggested that impaired GH-mediated JAK2 activation might be present. Indeed, we were able to demonstrate diminished JAK2 phosphorylation in CRF animals 10 minutes after $\mathrm{GH}$ injection. In the CRF rats phosphorylation of JAK2 in response to GH was quite variable; interestingly, the individual variation in JAK2 phosphorylation corresponded with that of the STAT proteins (Figure 3, b and d). Much of the variability of JAK2 phosphorylation was explained by differences in the degree of renal failure achieved; there was a strong correlation between the level of tyrosine phosphorylation and serum creatinine levels. Taken together, it appears that deficient JAK2 activation is the primary defect in GHR-related signal transduction in CRF.

As described above, GH-induced IGF-I expression was depressed in CRF. The molecular pathway leading to the induction of IGF-1 gene transcription by GH has not been elucidated. GH rapidly stimulates IGF-1 expression by a mechanism not requiring protein synthesis $(34,35)$. Evidence for the involvement of STAT5 in GH-induced IGF-1 synthesis has been provided by STAT5-knockout

Effects of 7-day GH treatment and CRF on hepatic CIS, SOCS-2, and SOCS-3 mRNA levels

\begin{tabular}{lcccccc}
\hline \multicolumn{4}{c}{ PF control } & \multicolumn{2}{c}{ CRF } & \\
& Vehicle & GH & Vehicle & GH & CRF effect & GH effect \\
CIS & $100 \pm 25^{\mathrm{A}}$ & $457 \pm 85^{\mathrm{B}}$ & $219 \pm 61^{\mathrm{A}}$ & $602 \pm 126^{\mathrm{B}}$ & NS & $P<0.0001$ \\
SOCS-2 & $100 \pm 26^{\mathrm{A}}$ & $482 \pm 17^{\mathrm{B}}$ & $222 \pm 68^{\mathrm{A}}$ & $909 \pm 137^{\mathrm{C}}$ & $P<0.05$ & $P<0.0001$ \\
SOCS-3 & $100 \pm 16^{\mathrm{A}}$ & $115 \pm 18^{\mathrm{A}}$ & $179 \pm 26^{\mathrm{A}}$ & $167 \pm 45^{\mathrm{A}}$ & $P<0.05$ & NS
\end{tabular}

Tissue was obtained 5-6 hours after the last GH injection. The mRNA levels were determined by RPA. Values were corrected for $18 \mathrm{~S}$ rRNA levels measured in the same assay. Data are expressed relative to PF vehicle mean value, which was assigned a value of 100 . Results are mean \pm SEM of three to seven rats per group. Superscript letters indicate significant differences between the groups within each row (NewmanKeuls test, $P<0.05$ ); groups with common superscript letters do not differ from each other. Results of two-way ANOVA are given in the two last columns. 
mice: circulating IGF- 1 levels are decreased in males deficient for the STAT5b isoform and in females if STAT5a and STAT5b genes are mutated $(14,15)$. Whereas no direct DNA-binding site for STATs has been found to date, GH-dependent IGF-1 transcription may be mediated by the cooperative action of the JAK-STAT pathway and one or more nuclear cofactors $(36,37)$. Hepatic IGFBP-1 expression is rapidly suppressed by GH (34) by a mechanism involving c-Fos and c-Jun (38). GH stimulates hepatic c-Fos transcription by binding of STAT1 and STAT3 homo-and heterodimers to a Sis-inducible element in the c-Fos promoter $(38,39)$. In addition, $G H$ activates c-Fos expression by stimulation of c-Raf and the MAP kinase pathway (10). The activation of both pathways requires phosphorylation of the GHR-associated kinase JAK2. Hence, the attenuated suppression of IGFBP-1 gene expression in GH-treated CRF rats, as well as the absent stimulation of IGF-1 expression in these animals, are compatible with diminished JAK2 and/or STAT activity in uremia.

Recently, a family of intracellular proteins has been defined that serve as intracellular negative feedback regulators of the JAK-STAT pathway by binding to cytokine receptor-JAK signaling complexes (11). Among the eight members of this family identified to date, $\mathrm{GH}$ stimulates the expression of SOCS-1, $-2,-3$, and CIS (11-13). When overexpressed in vitro, these $\mathrm{GH}$-inducible proteins partially or completely inhibit GH-dependent JAK2 activation (12). Whereas SOCS-1 and SOCS-3 appear to be stronger inhibitors than SOCS- 2 and CIS in transfection models, in vivo evidence for a role of SOCS in growth regulation has been provided for SOCS- 2 and CIS. Deletion of SOCS- 2 causes IGF-1 hyperexpression and gigantism in mice (16), whereas CIS overexpression causes severe growth retardation (40). To test whether altered expression of one of these inhibitors could be implicated as a potential cause of the depressed JAK2 phosphorylation, we measured mRNA levels of SOCS-2, SOCS-3, and CIS. We observed a wide variability of basal hepatic SOCS-2 expression, with a trend toward higher levels in CRF. However, after 1 week of GH treatment, SOCS-2 was clearly hyperexpressed in CRF animals and was more than twofold higher than in GH-treated controls. Interestingly, in the septic rat, another model of acquired GH resistance, treatment with GH also increases SOCS-2 mRNA levels (41). Basal SOCS-3 mRNA levels were modestly elevated in CRF. Whether the protein levels of these hyperexpressed genes are also increased in uremia remains to be established.

The mechanism for the increase in SOCS mRNA levels in the presence of impaired GH-activated JAK2-STAT signaling in uremia is not readily apparent. One potential process could involve the action of other members of the cytokine family (11), since several cytokines induce signal transduction by activating other members of the Janus kinase family such as JAK1, JAK3, and Tyk2 (42). Accordingly, if there were selective inhibition of JAK2 activation in the uremic state, it is conceivable that the increase in SOCS gene expression reflects cytokine sig- naling by way of a different Janus kinase. It is also possible that the increase in SOCS expression in uremia might be mediated by GH through a non-STAT-mediated pathway, for there is recent evidence, at least for SOCS-3, that nuclear STAT-binding sites are not essential for GH activation of the SOCS-3 promoter (43). Finally it should be kept in mind that the measured level of gene expression reflects the impact of uremia on several interacting processes including gene transcription and stability, cross-talk between signaling pathways, and the action of a variety of hormones and cytokines.

An acquired defect of GH-induced JAK-STAT signaling has been reported recently in rats with acute endotoxemia $(18,44)$. This manifests as a decrease in GH-induced JAK2 phosphorylation in the absence of a change in GHR abundance (18), diminished hepatic accumulation of nuclear STAT5P and STAT3P, and diminished binding of STAT5 to a GH-response element from the serine protease 2.1 inhibitor promoter (44). Interestingly, while the phosphorylation of the JAK2 protein was diminished, total JAK2P was unchanged because of an endotoxininduced increase in total Jak2 protein levels. These changes were accompanied by an acute increase in SOCS- 3 and CIS, and to a lesser degree SOCS- 2 expression, findings confirmed by others (45). These studies are supported by in vitro data that indicates that proinflammatory cytokines such as IL- 6 and IL- $1 \beta$ released during an endotoxin challenge may stimulate SOCS as part of an autoinhibitory intracellular feedback loop, causing concomitant GH resistance $(43,45,46)$. This is another example where sensitivity to some cytokines is maintained, despite resistance to GH. These reports may be relevant to the situation in patients with end-stage renal disease, for chronic subclinical inflammation is common in dialysis patients and appears to be a major cause of malnutrition and increased cardiovascular morbidity (47). It is tempting to speculate that persistent subclinical inflammation with proinflammatory cytokine release may contribute to the malnutrition of uremia by worsening the GH resistance in these patients by increasing SOCS protein production.

Taken together, we have defined a novel postreceptor mechanism of GH resistance in CRF. We found that despite a lack of change in GHR abundance, tyrosine phosphorylation of the GHR-associated tyrosine kinase JAK2 is diminished in uremia. This was associated with impaired phosphorylation and nuclear translocation of the downstream STAT-signaling proteins. Of note, expression of SOCS-2 and -3 mRNA levels were increased in CRF, and we propose that if accompanied by an increase in protein expression, then by suppressing JAK2 activity this may be a cause of the signaling defect. However, since regulation of the JAK-STAT pathway involves more than just the SOCS proteins, it is likely that several molecular mechanisms operative in uremia contribute to the impairment in GH signal transduction. These might include increased protein tyrosine phosphatase activity with more rapid dephosphorylation of the GHR and its signaling proteins, altered GH receptor and signaling 
protein degradation, and abnormal expression of novel signal regulatory proteins $(11,48)$. In conclusion, it appears that resistance to GH in uremia is not caused by a change in GHR number, but rather is the result of a defect in GH signal transduction. This signaling defect, together with resistance to IGF-I, likely contributes to the retarded body growth of children and the wasting that occurs in adults with renal failure.

\section{Acknowledgments}

This study was supported by grants from the Department Veterans Affairs (Merit Review grant to R. Rabkin), the NIH (grant 5R21 DK-53613 to R. Rabkin), and the Max Kade Foundation and the NIKI Foundation (to F. Schaefer).

1. Schaefer, F., and Mehls, O. 1998. Endocrine and growth disorders. In Pediatric nephrology. T.M. Barratt, E.D. Avner, and W.E. Harmon, editors. Williams \& Wilkins. Baltimore, Maryland, USA. 1197-1230.

2. Mehls, O., et al. 1988. Improvement of growth and food utilization by human recombinant growth hormone in uremia. Kidney Int. 33:45-52.

3. Chan,W., Valerie, K.C., and Chan, J.C.M. 1993. Expression of insulin-like growth factor-1 in uremic rats: growth hormone resistance and nutritional intake. Kidney Int. 43:790-795.

4. Tönshoff, B., et al. 1994. Reduced hepatic growth hormone (GH) receptor gene expression and increase in plasma $\mathrm{GH}$ binding protein in experimental uremia. Kidney Int. 45:1085-1092.

5. Tönshoff, B., et al. 1997. Decreased hepatic insulin-like growth factor (IGF)I and increased IGF binding protein-1 and -2 gene expression in experimental uremia. Endocrinology. 138:938-946.

6. Tönshoff, B., Blum, W.F., Wingen, A.M., and Mehls, O. 1995. Serum insulinlike growth factors (IGFs) and IGF binding proteins 1,2 and 3 in children with chronic renal failure: relationship to height and glomerular filtration rate. J. Clin. Endocrinol. Metab. 80:2684-2691.

7. Ding, H., Gao, X.L., Hirschberg, R., Vadgama, J.V., and Kopple, J.D. 1996. Impaired actions of insulin-like growth factor 1 on protein synthesis and degradation in skeletal muscle of rats with chronic renal failure. Evidence for a postreceptor defect. J. Clin. Invest. 97:1064-1075.

8. Tsao, T., Fervenza, F.C., Friedlaender, M., Chen, Y., and Rabkin, R. Effect of prolonged uremia on insulin-like growth I autophosphorylation and tyrosine kinase activity in muscle. Exp. Nephrol. In Press.

9. Villares, S.M., et al. 1994. Reduced food intake is the main cause of low growth hormone receptor expression in uremic rats. Mol. Cell. Endocrinol. 106:51-56.

10. Carter-Su, C., Rui, L, and Herington, J. 2000. Role of the tyrosine kinase JAK2 in signal transduction by growth hormone. Pediatr. Nephrol. 14:550-557.

11. Yasukawa, H., Sasaki, A., Yoshimura, A. 2000. Negative regulation of cytokine signaling pathways. Annu. Rev. Immunol. 18:143-164.

12. Ram, P.R., and Waxman, D.J. 1999. SOCS/CIS protein inhibition of growth hormone-stimulated STAT5 signaling by multiple mechanisms. J. Biol. Chem. 275:35553-35561.

13. Tollet-Egnell, P., Flores-Morales, A., Stavreus-Evers, A., Sahlin, L., and Norstedt, G. 1999. Growth hormone regulation of SOCS-2, SOCS-3, and CIS messenger ribonucleic acid expression in the rat. Endocrinology. 140:3693-3704.

14. Udy, G.B., et al. 1997. Requirement of STAT5b for sexual dimorphism of body growth rates and liver gene expression. Proc. Natl. Acad. Sci. USA. 94:7239-7244.

15. Teglund, S., et al. 1998. Stat5a and Stat5b proteins have essential and nonessential, or redundant, roles in cytokine responses. Cell. 93:841-850.

16. Metcalf, D., et al. 2000. Gigantism in mice lacking suppressor of cytokine signaling-2. Nature. 405:1069-1073.

17. Groesbeck, M.D., Parlow, A.F., and Daughaday, W.H. 1987. Stimulation of supranormal growth in prepubertal, adult plateaued, and hypophysectomized female rats by large doses of rat growth hormone: physiological effects and adverse consequences. Endocrinology. 120:1963-1975.

18. Mao,Y., et al. 1999. Endotoxin-induced inhibition of growth hormone receptor signaling in rat liver in vivo. Endocrinology. 140:5505-5515.

19. García, E., et al. 1997. Impaired secretion of growth hormone in experimental uremia: relevance of caloric deficiency. Kidney Int. 52:648-653.

20. Postel-Vinay, M.C. 1976. Binding of human growth hormone to rat liver membrane lactogenic and somatotropic sites. FEBS Lett. 69:137-140.

21. Loten, G.E., and Redshaw-Loten, J.C. 1986. Preparation of rat liver plasma membranes in a high yield. Anal. Biochem. 154:183-185.
22. Touster, O., Aronson, N.N.J., Dulaney, J.T., and Hendrickson, H. 1970. Isolation of rat liver plasma membranes. Use of nucleotide pyrophosphatase and phosphodiesterase I as marker enzymes. J. Cell. Biol. 47:604-618.

23. Fervenza, F.C., Tsao, T., and Rabkin, R. 1996. Response of the intrarenal insulin-like growth factor I axis to acute ischemic injury and treatment with growth hormone and epidermal growth factor. Kidney Int. 49:344-354.

24. Smith, W.C., Kuniyoshi, J., and Talamantes, F. 1989. Mouse serum growth hormone $(\mathrm{GH})$ binding protein has $\mathrm{GH}$ receptor extracellular and substituted transmembrane domains. Mol. Endocrinol. 3:984-990.

25. Shimasaki, S., and Ling, N. 1991. Identification and molecular characterization of insulin-like growth factor binding proteins (IGFBP-1, $-2,-3,-4,-5$ and -6). Prog. Growth Factor Res. 3:243-266.

26. Lowe, W.L., Jr., Lasky, S.R., Leroith, D., and Roberts, C.T., Jr. 1988. Distribution and regulation of rat insulin-like growth factor I messenger ribonucleic acids encoding alternative carboxyterminal E-peptides: evidence for differential processing and regulation in liver. Mol. Endocrinol. 2:528-535.

27. Gonzalez, I.L., and Schmickel, R.D. 1986. The human 18 S ribosomal RNA gene: evolution and stability. Am. J. Hum. Genet. 38:419-427.

28. Barnard, R., Bundesen, P.G., Rylatt, D.B., and Waters, M.J. 1985. Evidence from the use of monoclonal antibody probes for structural heterogeneity of the growth hormone receptor. Biochem J. 231:459-468.

29. Martínez, V., et al. 1999. Hepatic exression of growth hormone receptor/binding protein and insulin-like growth factor I genes in uremic rats. Influence of nutritional deficit. Growth Horm. IGF Res. 9:61-68.

30. Finidori, J., Postel-Vinay, M.C., and Kleinknecht, C. 1980. Lactogenic and somatotropic binding sites in liver membranes of rats with renal insufficiency. Endocrinology. 106:1960-1965.

31. Edmondson, S.R., et al. 2000. Growth hormone receptor abundance in tibial growth plates of uremic rats: GH/IGF-I treatment. Kidney Int. 58:62-70.

32. Tönshoff, B., et al. 1997. Reduced concentration of serum growth hormonebinding protein in children with chronic renal failure: correlation with $\mathrm{GH}$ insensitivity. J. Clin. Endocrinol. Metab. 82:1007-1013.

33. Powell, D., et al. 1997. Modulation of growth factors by growth hormone in children with chronic renal failure. Kidney Int. 51:1970-1979.

34. Gronowski, A.M., and Rotwein, P. 1995. Rapid changes in gene expression after in vivo growth hormone treatment. Endocrinology. 136:4741-4748.

35. Gronowski, A.M., Le Stunff, C., and Rotwein, P. 1996. Acute nuclear actions of growth hormone $(\mathrm{GH})$ : cycloheximide inhibits inducible activator protein-1 activity, but does not block GH-regulated signal transducer and activator of transcription activation or gene expression. Endocrinology. 137:55-64.

36. Benbassat, C., et al. 1999. Growth hormone-mediated regulation of insulinlike growth factor I promoter activity in C6 glioma cells. Endocrinology. 140:3073-3081.

37. Meton, I., Boot, P.J., Sussenbach, J.S., and Steenbergh, P.H. 1999. Growth hormone induces insulin-like growth factor-I gene transcription by a synergistic action of STAT5 and HNF-1alpha. FEBS Lett. 444:155-159.

38. Lu, X., Shi, Z., and Murphy, L.J. 1999. Growth hormone suppression of insulin-like growth factor binding protein-1 promoter activity. Mol. Cell. Endocrinol. 149:19-28.

39. Gronowski, A.M., et al. 1995. In vivo growth hormone treatment rapidly stimulates the tyrosine phosphorylation and activation of Stat3. Mol. Endocrinol. 9:171-177.

40. Matsumoto, A., et al. 1999. Suppression of STAT5 functions in liver, mammary glands, and T cells in cytokine-inducible SH2-containing protein 1 transgenic mice. Mol. Cell. Biol. 19:6396-6407.

41. Johnson, T.S., et al. 2001. Differential expression of suppressors of cytokine signaling genes in response to nutrition and growth hormone in the septic rat. J. Endocrinol. 169:409-415.

42. Touw, I.P., De Koning, J.P., Ward, A.C., and Hermans, M.H. 2000. Signaling mechanisms of cytokine receptors and their perturbances in disease. Mol. Cell. Endocrinol. 160:1-9.

43. Paul, C., Seiliez, I., Thissen, J.P., and Le Cam, A. 2000. Regulation of expression of the rat SOCS-3 gene in hepatocytes by growth hormone, interleukin6 and glucocorticoids mRNA analysis and promoter characterization. Eur. J. Biochem. 267:5849-5857.

44. Bergad, P.L., et al. 2000. Inhibition of growth hormone action in models of inflammation. Am. J. Physiol. Cell Physiol. 279:C1906-C1917.

45. Colson, A., Le Cam, A., Maiter, D., Edery, M., and Thissen, J.P. 2000. Potentiation of growth hormone-induced liver suppressors of cytokine signaling messenger ribonucleic acid by cytokines. Endocrinology. 141:3687-3695.

46. Boisclair, Y.R., Wang, J., Shi, J., Hurst, K.R., and Ooi, G.T. 2000. Role of the Suppressor of Cytokine Signaling-3 in mediating the inhibitory effects of interleukin-1 beta on the growth hormone-dependent transcription of the acid-labile subunit gene in liver cells. J. Biol. Chem. 275:3841-3847.

47. Kaysen, G.A. 1999. Inflammation, nutritional state and outcome in end stage renal disease. Miner. Electrolyte Metab. 25:242-250.

48. Carter-Su, C., Rui, L., and Stofega, M.R. 2000. SH2-B and SIRP: JAK2 binding proteins that modulate the actions of growth hormone. Rec. Prog. Horm. Res. 55:293-311. 